\title{
Avaliação Genética de Touros Usando Produção em Lactações Completas ou Parciais Projetadas. 1. Estimativa de Parâmetros Genéticos ${ }^{1}$
}

\author{
Cláudio Manoel Rodrigues de Melo², Antonio Ilson Gomes de Oliveira ${ }^{3}$, Mário Luiz Martinez ${ }^{4}$, \\ Rui da Silva Verneque ${ }^{4}$, Tarcisio de Moraes Gonçalves ${ }^{5}$, Rilke Tadeu Fonseca de Freitas ${ }^{6}$
}

\footnotetext{
RESUMO - Para calcular parâmetros genéticos e fenotípicos de lactações completas e parciais projetadas, foram utilizadas 4595 lactações de 2254 vacas, filhas de 145 touros e de 1618 matrizes, distribuídas em 18 rebanhos, com partos observados entre 1980 e 1997. A partir de 91, 151, 211 ou 241 dias de lactação, projetaram-se 10, 30, 50 ou 70\% das lactações, para a duração da lactação observada e para 305 dias. Os parâmetros genéticos foram obtidos utilizando o sistema MTDFREML. Independente da característica, incluíramse no modelo efeitos fixos de rebanho-ano, época de parto e idade da vaca ao parto, com termos linear e quadrático, considerando-se efeitos aleatórios de animal, efeito permanente de ambiente e erro. A estimativa da herdabilidade da produção de leite até 305 dias e o respectivo erro-padrão (P305d) foi de 0,17 (0,047) e das produções estimadas (PE), pela projeção de uma porcentagem das lactações, variaram de $0,17(0,048)$ a $0,27(0,056)$. As estimativas das correlações genéticas e fenotípicas e os respectivos erros-padrão entre P305d e as PE variaram de $0,94(0,466)$ a $1,00(0,589)$ e de $0,74(0,236)$ a $1,00(0,194)$, respectivamente. Concluiu-se que as herdabilidades das produções estimadas pela projeção de $10 \%$ das lactações, a partir de 91,151 e 211 dias, ou pela projeção de 10, 30, 50 ou 70\% das lactações, a partir de 241 dias, foram semelhantes àquela obtida para a produção de leite até 305 dias, e as produções de leite estimadas pela projeção das lactações foram altamente correlacionadas com a produção de leite até 305 dias.
}

Palavras-chave: correlação genética, herdabilidade, lactações parciais projetadas, máxima verossimilhança restrita, modelo animal, raça Gir

\section{Sires Genetic Evaluations Using Complete or Partial Projected Lactation Records. 1. Genetic Parameters Estimates}

\begin{abstract}
Records of 4,595 lactations from 2,254 cows, daughters of 145 sires and 1,618 dams of Gyr breed from 18 herds, with observed calving time from 1980 to 1997, were used to estimate the genetic and phenotypic parameters of milk yield up to 305 days and projected partial lactations. From 91, 151, 211 or 241 days in milk, 10, 30, 50 or $70 \%$ of the milk yield were projected for the observed and 305 days of lactation. The genetic parameters were obtained using MTDFREML system. Independent of the trait, as fixed effects the herd-year, calving season and calving age of the cow were included in the animal model, with linear and quadratic effects and it was considered as random effects of animal, the permanent environment and error. Heritability estimates for milk yield up to 305 days (P305d) was 0.17 (0.047), and for the estimated projected production (PE) of one percentage of lactations, ranged from 0.17 (0.048) to 0.27 (0.056). Genetic and phenotypic correlations estimates between P305d and PE ranged from 0.94 (0.466) to $1(0.589)$ and from 0.73 (0.236) to 1 (0.194), respectively. The production estimated by projecting the milk productions of $10 \%$ of the lactations from 91,151 and 211 days or by the projection of 10,30 , 50 or $70 \%$ of the lactations from the 241 days were similar to that obtained for the milk production up to 305 days, and that the milk productions estimated by the projections of the milk productions were highly correlated with the milk productions up to 305 days.
\end{abstract}

Key Words: animal model, genetic correlations, Gyr cattle, heritability, partial projected lactation, restricted maximum likelihood

\section{Introdução}

A seleção de bovinos para produção de leite tem sido baseada na análise da produção de leite até 305 dias de lactação. Entretanto, a utilização de lactações parciais nos programas de seleção de vacas e touros pode aumentar o número de filhas por touros e reduzir o intervalo de geração, o custo da obtenção de dados e a permanência de vacas de baixo valor genético no rebanho, já que a utilização desta informação pode reduzir em três a sete meses o tempo para a seleção dos animais superiores.

A viabilidade de se usarem produções de lactações parciais projetadas, na avaliação do mérito genético

\footnotetext{
${ }^{1}$ Parte da Dissertação de Mestrado em Zootecnia.

2 Doutorando em Zootecnia - USP.

${ }^{3}$ Professor Titular Aposentado da UFLA - Bolsista do CNPq. e.mail:tarcisio@ufla.br

${ }^{4}$ Pesquisador da Embrapa Gado de Leite.

5 Professor Assistente da UFLA. e.mail: tarcisio@ufla.br

${ }^{6}$ Professor Adjunto da UFLA. e.mail: rilke@ufla.br
} 
dos animais depende da confiabilidade das avaliações de touros obtidas por lactações parciais, das correlações genética e fenotípica entre a produção de lactações parciais e a produção de lactações até 305 dias e, finalmente, da herdabilidade da produção de lactações parciais.

Se as estimativas de herdabilidade da produção de leite de lactações parciais não forem muito diferentes das estimativas obtidas para a produção de leite de lactações completas e, desde que as estimativas das correlações entre estas produções sejam altas, então a ordem dos touros na avaliação usando registros de produções parciais, teoricamente, será a mesma da avaliação usando registros de produção até 305 dias de lactação, indicando a possibilidade de se usarem lactações parciais na seleção dos animais (TANDON e HARVEY, 1984; RIBAS e PEREZ, 1990).

Nesse contexto, vários trabalhos, em sua grande maioria utilizando registros de produções de animais da raça Holandês, têm descrito altas correlações, genética e fenotípica, entre a produção de lactações parciais e a produção aos 305 dias de lactação.

Os trabalhos têm mostrado que, de maneira geral, as estimativas de herdabilidade das produções de lactações parciais são semelhantes àquelas obtidas para a produção até 305 dias (TANDON e HARVEY, 1984; GENGLER, KEOWN e VAN VLECK, 1995; e WILMINK, 1987).

Produções de leite de lactações em progresso, projetadas para 305 dias, são altamente correlacionadas com a produção aos 305 dias de lactação. Estimativas de correlações genética de 0,85; 0,96; 0,99; e 1,00 para a produção aos 60,120 , 180 e 240 dias de lactação, projetadas para 305 dias, foram reportadas por WILMINK (1987), analisando as primeiras lactações de vacas holandesas. Estimativas similares foram obtidas por VAN VLECK (1964), TANDON e HARVEY (1984), SETHI e JAIN (1993), GENGLER, KEOWN e VAN VLECK (1995) e ZAHED, KHALIL e SOLIMAN (1997).

Correlações fenotípicas foram estimadas de registros mensais de várias lactações de vacas da raça Holandês por KEOWN e VAN VLECK (1971), que observaram estimativas variando de 0,65 a 0,90 , sendo os maiores valores observados entre 120 e 150 dias de lactação. Vários outros estudos constataram altas estimativas de correlações fenotípicas das produções de leite no dia do controle com a produção padronizada aos 305 dias (WILMINK, 1987; PANDER, HILL e THOMPSON, 1992; e PANDER e HILL, 1993) e aos 244 dias de lactação (RIBAS,
PEREZ e GUZMAN, 1994).

Os resultados acima demostram que o momento mais adequado para projeção da lactação se encontra entre o quinto e o sexto controle, parecendo, assim, não serem necessários mais que 150 dias de produção para que a lactação possa ser usada na avaliação do mérito genético dos animais.

O objetivo deste trabalho foi estimar parâmetros genéticos e fenotípicos para produção de leite até 305 dias e produções parciais projetadas.

\section{Material e Métodos}

Os dados utilizados neste estudo são provenientes de rebanhos da raça Gir, que participam do programa de melhoramento dessa raça, coordenado pela Embrapa Gado de Leite, em convênio com a Associação Brasileira dos Criadores de Gir Leiteiro (ABCGIL).

Após eliminar informações indesejáveis ao estudo (lactações consideradas anormais; lactações com produção zero em controles mensais; lactações com duração da lactação menor que 90 dias; lactações cujas vacas apresentaram idade ao primeiro parto menor que 18 meses; vacas que não apresentavam a primeira lactação; lactações de ordem de parto superior a cinco; e lactações de vacas sabidamente tratadas com Somatotropina Bovina Recombinante - bSTR) e considerando-se, ainda, um mínimo de cinco filhas por touro e de duas lactações por subclasse de rebanho-ano (RA), distribuídas nas duas estações de parição consideradas ( 1 = águas, outubro a março e 2 = seca, abril a setembro), analisaram-se 4595 lactações de 2254 vacas, filhas de 145 touros e de 1618 matrizes, distribuídas em 18 rebanhos, com partos ocorridos nos anos de 1980 a 1997.

As produções acumuladas foram calculadas até 90, 150, 210, 240 e 305 dias, usando o método do intervalo de teste, segundo EVERETT e CARTER (1968), e de uso oficial pelo Ministério da Agricultura (BRASIL, 1986).

A partir de 91, 151, 211 ou 241 dias de lactação, projetaram-se, aleatoriamente, $10,30,50$ ou $70 \%$ das lactações, tanto para a duração da lactação observada, como para 305 dias, utilizando-se funções quadráticas logarítmicas obtidas por GONÇALVES, MARTINEZ e MILAGRES (1997).

Para classificação das vacas quanto ao nível de produção, utilizou-se a média da produção de leite na primeira lactação. Assim, o nível baixo de produção compreende todas as vacas que apresentaram produ- 
ção média de leite, na primeira lactação, inferior a $2.230 \mathrm{~kg}$ de leite (média da produção de leite na primeira lactação), e nível alto de produção as demais.

Para projeção da lactação a partir de 91, 151 e 211 dias de lactação, utilizaram-se as seguintes equações:

$$
\hat{\mathbf{y}}=-1,19-0,084 t+0,000129 t^{2}+3,24 \operatorname{lnt}, \text { para }
$$

baixo nível de produção da vaca, e

$$
\hat{\mathbf{y}}=11,09+0,002 \mathrm{t}-0,0000138 \mathrm{t}^{2}-0,031 \mathrm{lnt}, \text { para }
$$

alto nível de produção da vaca.

Para projeção da lactação a partir de 241 dias de lactação, utilizaram-se as seguintes equações:

$$
\hat{\mathbf{y}}=1,07-0,054 \mathrm{t}+0,000082 \mathrm{t}^{2}+2,23 \ln \mathrm{t}, \quad \text { para }
$$

baixo nível de produção da vaca, e

$$
\hat{\mathbf{y}}=14,17-0,006 \mathrm{t}-0,000055 \mathrm{t}^{2}-0,591 \mathrm{nt}, \text { para }
$$

alto nível de produção da vaca, sendo $\hat{\mathbf{y}}$ a estimativa da produção de leite diária, em quilogramas, e t o período de tempo desde o parto, em dias. A produção no período projetado é dada por: $\sum_{i=1}^{t} \hat{\mathbf{y}}_{\mathrm{i}}$, em que $\hat{\mathbf{y}}_{\mathbf{i}}$ é a produção estimada no dia i e t, como definido acima.

Além da característica produção de leite até 305 dias (P305d), foram geradas outras 32 estimativas de produção, conforme mostrado na Tabela 1 .

Os dados foram preparados utilizando-se o pacote computacional "SAS ${ }^{\circledR} "$ (STATISTICAL ANALYSIS SYSTEM) versão 6.12 (1996).
Para a análise utilizou-se o seguinte modelo estatístico:

$$
\mathbf{y}=\mathbf{X} \underset{\sim}{\boldsymbol{\beta}}+\underset{\sim}{\mathbf{Z}}+\mathbf{W} \underset{\sim}{\mathbf{c}}+\underset{\sim}{\mathbf{e}}
$$

em que

y é o vetor das variáveis dependentes;

$\mathbf{X}$ é a matriz de incidência dos efeitos fixos;

$\boldsymbol{\beta}$ é o vetor de efeitos fixos (rebanho-ano, época de parto, idade da vaca ao parto com termos linear e quadrático);

$\mathbf{Z}$ é a matriz de incidência dos efeitos genéticos diretos;

$\underset{\sim}{\mathbf{a}}$ é o vetor dos efeitos aleatórios dos valores genéticos diretos do animal;

W é a matriz de incidência dos efeitos permanente de ambiente;

c é o vetor dos efeitos permanentes de ambiente; e

é é o vetor dos erros aleatórios associados a cada observação.

Assumiu-se que $\underset{\sim}{\mathbf{y}}, \underset{\sim}{\mathbf{a}}, \underset{\sim}{\mathbf{c}}$ e $\underset{\sim}{\mathbf{e}}$ possuem distribuição conjunta normal multivariada, como apresentado a seguir.

\begin{tabular}{|c|c|c|c|c|}
\hline \multicolumn{2}{|c|}{$\begin{array}{l}\text { Produções estimadas } \\
\text { Estimated yields }\end{array}$} & \multirow[b]{2}{*}{$\begin{array}{c}\text { \% de lactações } \\
\text { projetadas } \\
\% \text { of extended } \\
\text { lactations }\end{array}$} & \multirow[b]{2}{*}{$\begin{array}{l}\text { Projetadas a partir de } \\
\text { Extended starting from }\end{array}$} & \multirow[b]{2}{*}{$\begin{array}{l}\text { Resultado da projeção } \\
\text { somado à produção } \\
\text { calculada até } \\
\text { Extension results added } \\
\text { to the calculated up to }\end{array}$} \\
\hline $\begin{array}{l}\text { Pela projeção } \\
\text { para a DL } \\
\text { Extension to } L L\end{array}$ & $\begin{array}{c}\text { Pela projeção } \\
\text { para } 305 \text { dias } \\
\text { Extension to } \\
305 \text { days }\end{array}$ & & & \\
\hline P090d10\%DL & P090d10\% & $10 \%$ & $\begin{array}{l}91 \text { dias de lactação } \\
91 \text { days of lactation }\end{array}$ & $\begin{array}{l}90 \text { dias de lactação } \\
90 \text { days of lactation }\end{array}$ \\
\hline P090d30\%DL & P090d30\% & $30 \%$ & 91 dias de lactação & 90 dias de lactação \\
\hline P090d50\%DL & P090d50\% & $50 \%$ & 91 dias de lactação & 90 dias de lactação \\
\hline P090d70\%DL & P090d70\% & $70 \%$ & 91 dias de lactação & 90 dias de lactação \\
\hline P150d10\%DL & $\mathrm{P} 150 \mathrm{~d} 10 \%$ & $10 \%$ & 151 dias de lactação & 150 dias de lactação \\
\hline P150d30\%DL & $\mathrm{P} 150 \mathrm{~d} 30 \%$ & $30 \%$ & 151 dias de lactação & 150 dias de lactação \\
\hline P150d50\%DL & P090d50\% & $50 \%$ & 151 dias de lactação & 150 dias de lactação \\
\hline P150d70\%DL & $\mathrm{P} 150 \mathrm{~d} 70 \%$ & $70 \%$ & 151 dias de lactação & 150 dias de lactação \\
\hline P210d10\%DL & $\mathrm{P} 210 \mathrm{~d} 10 \%$ & $10 \%$ & 211 dias de lactação & 210 dias de lactação \\
\hline P210d $30 \% D L$ & $\mathrm{P} 210 \mathrm{~d} 30 \%$ & $30 \%$ & 211 dias de lactação & 210 dias de lactação \\
\hline P210d50\%DL & $\mathrm{P} 210 \mathrm{~d} 50 \%$ & $50 \%$ & 211 dias de lactação & 210 dias de lactação \\
\hline $\mathrm{P} 210 \mathrm{~d} 70 \% \mathrm{DL}$ & $\mathrm{P} 210 \mathrm{~d} 70 \%$ & $70 \%$ & 211 dias de lactação & 210 dias de lactação \\
\hline P240d10\%DL & $\mathrm{P} 240 \mathrm{~d} 10 \%$ & $10 \%$ & 241 dias de lactação & 240 dias de lactação \\
\hline P240d30\%DL & $\mathrm{P} 240 \mathrm{~d} 30 \%$ & $30 \%$ & 241 dias de lactação & 240 dias de lactação \\
\hline P240d50\%DL & $\mathrm{P} 240 \mathrm{~d} 50 \%$ & $50 \%$ & 241 dias de lactação & 240 dias de lactação \\
\hline $\mathrm{P} 240 \mathrm{~d} 70 \% \mathrm{DL}$ & $\mathrm{P} 240 \mathrm{~d} 70 \%$ & $70 \%$ & 241 dias de lactação & 240 dias de lactação \\
\hline
\end{tabular}

Tabela 1 - Descrição das produções estudadas, em que uma porcentagem das lactações foi projetada para a duração da lactação observada (DL) ou para 305 dias

Table 1 - Description of the studied productions where a percentage of the lactations were projected for the length of the lactation observed (LL) or for 305 days 


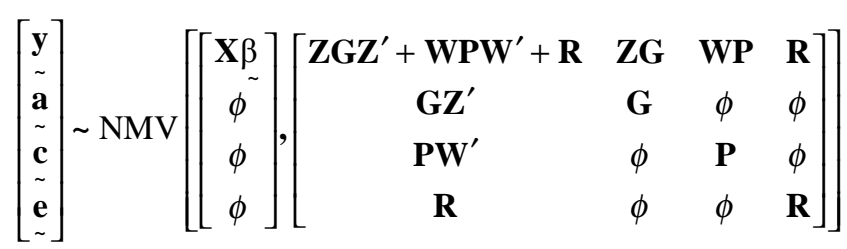

em que $\mathbf{G}=\mathbf{A} \sigma_{\mathrm{a}}^{2}, \quad \mathbf{P}=\mathbf{I}_{\mathrm{p}} \sigma_{\mathrm{c}}^{2}$ e $\mathbf{R}=\mathbf{I} \sigma_{\mathrm{e}}^{2}$ ou $\mathbf{G}=\mathbf{A} \otimes \mathbf{G}_{0}, \mathbf{P}=\mathbf{I}_{\mathrm{p}} \otimes \mathbf{P}_{0}$ e $\mathbf{R}=\mathbf{I} \otimes \mathbf{R}_{0}$, quando se trata de análise de uma ou mais características, respectivamente, em que $\mathbf{A}$ é a matriz, de ordem m, de coeficientes de parentesco entre os animais avaliados; $\mathbf{Z}$, a matriz, de ordem $\mathrm{n} \times \mathrm{m}$, de incidência dos valores genéticos; W, a matriz, de ordem $\mathrm{n} \times \mathrm{p}$, de incidência dos efeitos permanentes de ambiente; $\mathbf{I}$, uma matriz identidade, de ordem $\mathrm{n} ; \mathbf{I}_{\mathrm{p}}$, uma matriz identidade, de ordem p; $\mathbf{G}_{0}$, a matriz, de ordem q, de variâncias e covariâncias genética aditiva; $\mathbf{P}_{0}$, a matriz, de ordem q, de variâncias e covariâncias dos efeitos permanentes de ambiente; $\mathbf{R}_{0}$, a matriz, de ordem $\mathbf{q}$, de variâncias e covariâncias dos resíduos; $\sigma_{\mathrm{a}}^{2}$, a variância genética aditiva; $\sigma_{\mathrm{c}}^{2}$, a variância de efeito permanente de ambiente; $\sigma_{\mathrm{e}}^{2}$, a variância de efeito temporário de ambiente, sendo que $n$ é o número total de observações; $p$, o número de indivíduos com observações; $m$, o número de indivíduos da população (com ou sem informações); e q, o número de características em análise.

As herdabilidades, repetibilidades e correlações foram estimadas por meio dos componentes de (co)variâncias genéticos, permanente de ambiente, temporário de ambiente e fenotípico, obtidos no sistema MTDFREML (BOLDMAN et al., 1995), no qual os dados são analisados sob modelo animal (VAN VLECK, 1992) e o método da máxima verossimilhança restrita é usado para cálculo dos componentes de covariância. $\mathrm{O}$ critério de convergência estabelecido foi que a variância dos n valores da função $(-2 \log \Lambda)$ do SIMPLEX fosse menor que $10^{-9}$, sendon igual ao número de componentes de covariância a serem estimados.

\section{Resultados e Discussão}

\section{Parâmetros genéticos}

\section{Herdabilidade e repetibilidade}

A estimativa da média da produção de leite até 305 dias (P305d) foi de 2445,75 kg (13,35), com coeficiente de variação de $37,35 \%$; as médias para as produções estimadas (PE) para duração da lactação observada variaram de $2381,98(12,48)$ a 2458,44
$(13,64)$ e as estimativas para as lactações projetadas (PE) para 305 dias, de 2407,43 (12,43) a 2544,68 (13,56).

Verificaram-se valores semelhantes para as médias da produção de leite das diferentes características consideradas, sendo as médias das produções estimadas pela projeção de 10 ou $30 \%$ das lactações mais semelhantes à média para P305d.

Constatou-se que, embora as PE sejam semelhantes à $\mathrm{P} 305 \mathrm{~d}$, algumas lactações poderiam estar sendo super ou subestimadas, em valores expressivos, pela projeção, contudo estas diferenças se anularam na média, não tendo sido constatadas pelas diferenças entre as PE e P305d. A duração da lactação média nos dados em estudo foi de 292,41 $(0,94)$ dias.

Pelas estimativas de herdabilidade $\left(\hat{h}^{2}\right)$, repetibilidade $(\hat{\mathrm{t}})$ e seus respectivos erros-padrão (EP) obtidos neste estudo (Tabela 2), observa-se que os valores de $\hat{\mathrm{h}}^{2}$ e $\hat{\mathrm{t}}$ das PE, a partir de 211 ou 241 dias de lactação, foram mais semelhantes aos obtidos para P305d do que as estimativas obtidas com base nas PE a partir de 91 ou 151 dias de lactação.

As PE pela projeção de 10 ou $30 \%$ das lactações foram as que apresentaram estimativas de herdabilidade e repetibilidade mais semelhantes às obtidas para a $\mathrm{P} 305 \mathrm{~d}$, resultado já esperado, tendo em vista que foram projetadas poucas lactações, sendo, assim insuficiente para alterar significativamente estas produções, quando comparadas à $\mathrm{P} 305 \mathrm{~d}$.

Estimativas de $0,17(0,047)$ e $0,50(0,047)$ para a herdabilidade e a repetibilidade, respectivamente, foram obtidas para P305d, considerada como característica padrão, por ser utilizada em grande parte dos trabalhos de avaliação genética de bovinos leiteiros.

Estimativas mais elevadas obtidas para as PE pela projeção de 50 ou $70 \%$ das lactações podem ser explicadas, em parte, pelo fato de que maior número de registros novos foi gerado nestes casos, o que parece ter levado à redução na variância ambiental e aumento na variância genética aditiva.

As estimativas de $\hat{\mathrm{h}}^{2}$ obtidas neste trabalho assemelham-se às obtidas por TANDON e HARVEY (1984) e SETHI e JAIN (1993), para produções de lactações "truncadas" em diferentes estádios da lactação, e WILMINK (1987), em que todas as lactações foram projetadas a partir de diferentes estádios da lactação, para 305 dias.

Em animais da raça Gir, estimativas de 0,25 $(0,047), 0,37(0,056), 0,12(0,04)$ e $0,24(0,06)$ foram obtidas por RAMOS (1984), SOUZA (1991), VERNEQUE (1994) e VERNEQUE et al. (1998), respectivamente, sendo as duas primeiras pelo méto- 
Rev. bras. zootec.

Tabela 2 - Estimativas da herdabilidade $\left(\hat{\mathrm{h}}^{2}\right)$, repetibilidade $(\hat{\mathrm{t}})$ e respectivos erros-padrão (EP) da produção de leite até 305 dias de lactação (P305d) e das produções estimadas a partir da projeção de uma porcentagem das lactações para a duração da lactação observada e para 305 dias, obtidas por análise univariada

Table 2 - Estimates of the heritability $\left(\hat{h}^{2}\right)$, repeatability $(\hat{t})$ and respective standard errors (SE) of milk yield up to 305 days of lactation (P305d) and of the yields estimated from the projection of a percentage of the lactations for the length of observed lactation and for 305 days, obtained for univariate analysis

\begin{tabular}{|c|c|c|c|c|c|}
\hline $\begin{array}{l}\text { Produções } \\
\text { estimadas } \\
\text { Estimated } \\
\text { yields }\end{array}$ & $\hat{\mathrm{h}}^{2}(\mathrm{EP})$ & $\hat{\mathrm{t}}(\mathrm{EP})$ & $\begin{array}{c}\text { Produções } \\
\text { estimadas } \\
\text { Estimated } \\
\text { yields }\end{array}$ & $\hat{\mathrm{h}}^{2}(\mathrm{EP})$ & $\hat{\mathrm{t}}(\mathrm{EP})$ \\
\hline P305d & $0,17(0,047)$ & $0,50(0,047)$ & - & - & - \\
\hline P090d10\%DL & $0,18(0,048)$ & $0,51(0,047)$ & P090d10\% & $0,18(0,047)$ & $0,50(0,047)$ \\
\hline P090d30\%DL & $0,21(0,051)$ & $0,52(0,050)$ & P090d30\% & $0,20(0,050)$ & $0,52(0,049)$ \\
\hline P090d50\%DL & $0,25(0,056)$ & $0,58(0,055)$ & P090d50\% & $0,24(0,054)$ & $0,58(0,053)$ \\
\hline P090d70\%DL & $0,22(0,055)$ & $0,66(0,054)$ & P090d70\% & $0,23(0,056)$ & $0,68(0,055)$ \\
\hline P150d10\%DL & $0,17(0,047)$ & $0,50(0,047)$ & P150d10\% & $0,18(0,048)$ & $0,50(0,047)$ \\
\hline P150d30\%DL & $0,21(0,052)$ & $0,54(0,051)$ & P150d30\% & $0,23(0,053)$ & $0,55(0,052)$ \\
\hline P150d50\%DL & $0,25(0,056)$ & $0,58(0,055)$ & P150d50\% & $0,27(0,057)$ & $0,58(0,056)$ \\
\hline P150d70\%DL & $0,23(0,055)$ & $0,63(0,054)$ & P150d70\% & $0,26(0,059)$ & $0,66(0,058)$ \\
\hline P210d10\%DL & $0,17(0,047)$ & $0,50(0,047)$ & P210d10\% & $0,18(0,048)$ & $0,51(0,048)$ \\
\hline $\mathrm{P} 210 \mathrm{~d} 30 \% \mathrm{DL}$ & $0,20(0,051)$ & $0,53(0,050)$ & $\mathrm{P} 210 \mathrm{~d} 30 \%$ & $0,21(0,052)$ & $0,53(0,051)$ \\
\hline $\mathrm{P} 210 \mathrm{~d} 50 \% \mathrm{DL}$ & $0,23(0,053)$ & $0,56(0,053)$ & $\mathrm{P} 210 \mathrm{~d} 50 \%$ & $0,23(0,054)$ & $0,56(0,053)$ \\
\hline $\mathrm{P} 210 \mathrm{~d} 70 \% \mathrm{DL}$ & $0,22(0,053)$ & $0,58(0,052)$ & $\mathrm{P} 210 \mathrm{~d} 70 \%$ & $0,24(0,056)$ & $0,60(0,055)$ \\
\hline P240d10\%DL & $0,17(0,047)$ & $0,49(0,047)$ & P240d10\% & $0,17(0,047)$ & $0,50(0,047)$ \\
\hline P240d30\%DL & $0,17(0,047)$ & $0,51(0,047)$ & P240d30\% & $0,17(0,047)$ & $0,49(0,047)$ \\
\hline P240d50\%DL & $0,18(0,048)$ & $0,49(0,047)$ & P240d50\% & $0,17(0,048)$ & $0,49(0,047)$ \\
\hline $\mathrm{P} 240 \mathrm{~d} 70 \% \mathrm{DL}$ & $0,19(0,049)$ & $0,50(0,048)$ & P240d70\% & $0,18(0,048)$ & $0,49(0,048)$ \\
\hline
\end{tabular}

do dos quadrados mínimos e as demais pelo REML, sob modelo animal. Estimativa superior à deste trabalho, utilizando REML sob modelo de touro, foi descrita por PANDER et al. (1992), em registros de vacas da raça Holandês $\left(\hat{\mathrm{h}}^{2}=0,49\right)$, sendo que KEOWN e VAN VLECK (1971), AURAN (1976), DANELL (1982), WILMINK (1987), RIBAS e PEREZ (1990) e RIBAS et al. (1994) obtiveram valores mais próxi$\operatorname{mos}(0,25 ; 0,25 ; 0,30 ; 0,31 ; 0,29$; e 0,29 , respectivamente), também para vacas da raça Holandês.

A menor estimativa obtida no presente trabalho pode ter sido causada, especialmente, pela grande variabilidade nos registros de produção de leite da população estudada, sendo que a maior parte desta variabilidade, possivelmente, seja devida a fatores ambientais.

As estimativas de herdabilidade $\left(\hat{h}^{2}\right)$ e repetibilidade (t) e os respectivos erros-padrão (EP) da produção de leite até $305 \mathrm{~d}$ e das produções de leite estimadas, obtidas por análise bivariada, mostraram que os valores obtidos para as PE a partir de 241 dias, foram mais semelhantes às obtidas para P305d.

As estimativas de herdabilidade e repetibilidade obtidas para P305d, oriundas das análises uni e bivariada, foram semelhantes, tendo em vista que as médias das estimativas de herdabilidade e repetibilidade, da produção de leite até 305 dias, obtidas por análise bivariada, foram de $0,19(0,048)$ e $0,52(0,047)$, enquanto as estimativas obtidas por análise univariada foram $0,17(0,047)$ e $0,50(0,047)$, respectivamente.

Estimativas de repetibilidade de $0,45(0,05)$ para produção de leite até 90 dias; $0,46(0,05)$ para produção até 150 dias; $0,47(0,05)$ para produção até 210 dias; e 0,46 $(0,05)$ para produção até 305 dias de lactação foram reportadas por VERNEQUE et al. (1998), obtidas pelo método REML em registros de vacas da raça Gir. Trabalhos anteriores de SOUZA (1991), pelo método dos quadrados mínimos, e VERNEQUE (1994), pelo método REML, relataram estimativas de $0,40(0,019)$ e 0,46 para produção de leite até 305 dias, em registros de produção de leite de vacas da raça Gir.

\section{Coeficientes de correlação}

\section{Correlação genética}

É possível constatar, por intermédio das Tabelas 3 e 4 , que as estimativas dos coeficientes de correlação genética entre as produções de leite estimadas pela projeção de uma porcentagem das lactações e 
MELO et al.

Tabela 3 - Estimativas das correlações genéticas $\left(\hat{r}_{a}\right)$, do efeito permanente de ambiente $\left(\hat{r}_{\mathrm{c}}\right)$ e do efeito temporário de ambiente $\left(\hat{\mathrm{r}}_{\mathrm{e}}\right)$, respectivos errospadrão (EP) e correlação fenotípica $\left(\hat{r}_{p}\right)$ entre a produção de leite até 305 dias (P305d) e as produções estimadas pela projeção de uma porcentagem das lactações para duração da lactação observada

Table 3 - Estimates of the genetic correlations $\left(\hat{\mathrm{r}}_{\mathrm{a}}\right)$ of the permanent effect of environment $\left(\hat{\mathrm{r}}_{\mathrm{c}}\right)$ and the temporary effect of environment $\left(\hat{\mathrm{r}}_{\mathrm{e}}\right)$, respective standard errors $(E P)$ and phenotypic correlation ( $\hat{\mathrm{r}}_{\mathrm{p}}$ ) between milk yield up to 305 days (P305d) and the yields estimated by the projection of a percentage of the lactations for for the observed lactation length

\begin{tabular}{lcccc}
\hline $\begin{array}{l}\text { Produções estimadas } \\
\text { Estimated yields }\end{array}$ & $\hat{\mathrm{r}}_{\mathrm{a}}(\mathrm{EP})$ & $\hat{\mathrm{r}}_{\mathrm{c}}(\mathrm{EP})$ & $\hat{\mathrm{r}}_{\mathrm{e}}(\mathrm{EP})$ & $\hat{\mathrm{r}}_{\mathrm{p}}$ \\
\hline P090d10\%DL & $1,00(0,537)$ & $1,00(0,283)$ & $0,94(0,056)$ & 0,97 \\
P090d30\%DL & $1,00(0,515)$ & $0,96(0,283)$ & $0,85(0,051)$ & 0,91 \\
P090d50\%DL & $1,00(0,482)$ & $0,90(0,271)$ & $0,78(0,047)$ & 0,86 \\
P090d70\%DL & $0,94(0,483)$ & $0,86(0,226)$ & $0,75(0,046)$ & 0,82 \\
P150d10\%DL & $1,00(0,589)$ & $1,00(0,261)$ & $0,97(0,057)$ & 0,98 \\
P150d30\%DL & $1,00(0,518)$ & $0,98(0,284)$ & $0,91(0,054)$ & 0,95 \\
P150d50\%DL & $1,00(0,486)$ & $0,94(0,283)$ & $0,87(0,052)$ & 0,92 \\
P150d70\%DL & $0,97(0,492)$ & $0,91(0,249)$ & $0,86(0,051)$ & 0,97 \\
P210d10\%DL & $1,00(0,523)$ & $1,00(0,268)$ & $0,98(0,055)$ & 0,99 \\
P210d30\%DL & $1,00(0,532)$ & $0,99(0,286)$ & $0,97(0,058)$ & 0,98 \\
P210d50\%DL & $1,00(0,525)$ & $0,98(0,263)$ & $0,95(0,056)$ & 0,97 \\
P210d70\%DL & $0,99(0,501)$ & $0,97(0,281)$ & $0,94(0,015)$ & 0,96 \\
P240d10\%DL & $1,00(0,540)$ & $1,00(0,284)$ & $0,99(0,058)$ & 1,00 \\
P240d30\%DL & $1,00(0,539)$ & $1,00(0,282)$ & $0,98(0,057)$ & 0,99 \\
P240d50\%DL & $1,00(0,546)$ & $1,00(0,294)$ & $0,97(0,057)$ & 0,99 \\
P240d70\%DL & $1,00(0,539)$ & $1,00(0,265)$ & $0,98(0,055)$ & 0,99 \\
\hline
\end{tabular}

Tabela 4 - Estimativas das correlações genéticas $\left(\hat{r}_{a}\right)$, do efeito permanente de ambiente $\left(\hat{r}_{c}\right)$ e do efeito temporário de ambiente $\left(\hat{r}_{\mathrm{e}}\right)$, respectivos errospadrão (EP) e correlação fenotípica $\left(\hat{r}_{p}\right)$ entre a produção de leite até 305 dias (P305d) e as produções estimadas pela projeção de uma porcentagem das lactações para 305 dias

Table 4 - Estimates of the genetic correlations $\left(\hat{\mathrm{r}}_{\mathrm{a}}\right)$ of the permanent effect of environment $\left(\hat{\mathrm{r}}_{\mathrm{c}}\right)$ and the temporary effect of environment $\left(\hat{\mathrm{r}}_{\mathrm{e}}\right)$ respective standard errors $(E P)$ and phenotypic correlation ( $\hat{\mathrm{r}}_{\mathrm{p}}$ ) between milk yield up to 305 days (P305d) and the yields estimated by the projection of a percentage of the lactations for 305 days

\begin{tabular}{lcccc}
\hline $\begin{array}{l}\text { Produções estimadas } \\
\text { Estimated yields }\end{array}$ & $\hat{\mathrm{r}}_{\mathrm{a}}(\mathrm{EP})$ & $\hat{\mathrm{r}}_{\mathrm{c}}(\mathrm{EP})$ & $\hat{\mathrm{r}}_{\mathrm{e}}(\mathrm{EP})$ & $\hat{\mathrm{r}}_{\mathrm{p}}$ \\
\hline P090d10\% & $0,99(0,527)$ & $1,00(0,298)$ & $0,91(0,054)$ & 0,95 \\
P090d30\% & $1,00(0,513)$ & $0,95(0,272)$ & $0,78(0,047)$ & 0,88 \\
P090d50\% & $1,00(0,473)$ & $0,85(0,259)$ & $0,68(0,042)$ & 0,80 \\
P090d70\% & $0,94(0,466)$ & $0,78(0,205)$ & $0,60(0,038)$ & 0,73 \\
P150d10\% & $1,00(0,541)$ & $1,00(0,275)$ & $0,95(0,056)$ & 0,97 \\
P150d30\% & $1,00(0,495)$ & $0,97(0,295)$ & $0,87(0,052)$ & 0,93 \\
P150d50\% & $0,99(0,467)$ & $0,92(0,292)$ & $0,80(0,048)$ & 0,88 \\
P150d70\% & $0,95(0,462)$ & $0,89(0,251)$ & $0,76(0,046)$ & 0,84 \\
P210d10\% & $1,00(0,500)$ & $1,00(0,307)$ & $0,97(0,056)$ & 0,99 \\
P210d30\% & $1,00(0,500)$ & $0,99(0,312)$ & $0,94(0,056)$ & 0,97 \\
P210d50\% & $1,00(0,493)$ & $0,97(0,296)$ & $0,91(0,054)$ & 0,95 \\
P210d70\% & $0,97(0,487)$ & $0,96(0,275)$ & $0,89(0,053)$ & 0,93 \\
P240d10\% & $1,00(0,519)$ & $1,00(0,272)$ & $0,99(0,057)$ & 1,00 \\
P240d30\% & $1,00(0,535)$ & $1,00(0,280)$ & $0,98(0,057)$ & 0,99 \\
P240d50\% & $1,00(0,552)$ & $1,00(0,291)$ & $0,97(0,057)$ & 0,98 \\
P240d70\% & $1,00(0,551)$ & $1,00(0,291)$ & $0,96(0,057)$ & 0,98 \\
\hline
\end{tabular}


P305d foram altas e positivas.

Quando as lactações foram projetadas para a duração da lactação observada, as estimativas das correlações genéticas entre PE e P305d variaram de $0,94(0,483)$ a $1,00(0,589)$ e, no caso em que as lactações foram projetadas para 305 dias de lactação, de $0,94(0,466)$ a $1,00(0,541)$. Pela semelhança entre as estimativas, é possível constatar que não houve diferença entre projetar para duração da lactação observada ou para 305 dias, o que pode ser explicado pelo fato de a média de duração das lactações, 292,41 dias, ser próxima a 305 dias.

Observa-se que as estimativas obtidas para a correlação entre produção de leite até 305 dias (P305d) e PE pela projeção a partir de 211 ou 241 dias foram ligeiramente mais altas $(0,99$ a 1$)$ que as obtidas entre P305d e PE pela projeção a partir de 91 ou 151 dias (0,94 a 1). Estas diferenças foram maiores entre P305d e PE pela projeção de $70 \%$ das lactações a partir de 91 dias de lactação. Pode-se constatar tendência de valores pouco menores $(0,94$ a 0,97$)$ nas estimativas das correlações, com o aumento da porcentagem de lactações projetadas e do período de projeção (projeção de $70 \%$ das lactações a partir de 91 ou 151 dias de lactação).

As estimativas obtidas no presente trabalho são similares às encontradas na literatura: 0,$85 ; 0,96 ; 0,99$ e 1 entre P305d e as produções de lactações projetadas a partir de 60,120, 180 e 240 dias de lactação, respectivamente (WILMINK, 1987).

Estimativas também elevadas foram obtidas por TANDON e HARVEY (1984), SETHI e JAIN (1993) e VERNEQUE et al. (1998) entre a produção de leite de lactações parciais e a produção de leite até 305 dias.

\section{Correlação fenotípica}

Tal como as estimativas dos coeficientes de correlação genética, as estimativas dos coeficientes de correlação fenotípica foram altas e positivas (Tabelas 3 e 4).

Semelhantemente, também foram obtidas as menores estimativas entre P305d e as PE, por meio da projeção de $70 \%$ das lactações a partir de 91 dias de lactação (0,82 - entre P305d e P090d70\%DL e 0,73 - entre P305d e P090d70\%).

Nota-se, ainda, que quanto mais tardia for a projeção das lactações e menor a porcentagem de lactações projetadas, maiores serão as estimativas dos coeficientes de correlação, sugerindo, neste caso, resultados mais confiáveis nos testes de avaliação genética, quando lactações parciais são também utilizadas.

Pode-se observar que, à semelhança do que ocor- reu com as estimativas de correlação genética, no caso da correlação fenotípica, as estimativas tendem a ser maiores entre as PE pela projeção de uma porcentagem das lactações, para a duração da lactação observada, em comparação às estimativas obtidas das PE pela projeção das lactações para 305 dias.

A literatura é escassa em estudos nos quais é estimada a correlação fenotípica entre produção de leite de lactações parciais e P305d. Contudo, os poucos relatos encontrados na literatura mostram valores de correlação relativamente altos. Estimativas variando de 0,64 a 0,99 foram obtidas por AURAN (1976), entre produções de leite acumuladas aos 30, $60,90, \ldots, 300$ dias e a produção de leite aos 305 dias de lactação. Semelhantemente, estimativas variando de 0,69 a 0,$97 ; 0,65$ a 0,99 ; e 0,60 a 0,97 foram obtidas, respectivamente, por DANELL (1982), PANDER e HILL (1993) e RIBAS et al. (1994), entre as produções de leite acumuladas e a produção aos 305 dias ou aos 244 dias de lactação, no caso do último estudo. Estas estimativas são inferiores às do presente estudo, o que se deve, possivelmente, ao fato destes autores não terem projetado as lactações, e tão somente utilizado as produções acumuladas nos diferentes estágios da lactação estudados.

Correlação dos efeitos permanente e temporário de ambiente

Os coeficientes de correlação, tanto do efeito permanente $\left(\hat{r}_{c}\right)$ como temporário $\left(\hat{r}_{e}\right)$ de ambiente, foram próximos a um, seguindo tendência similar à obtida para os coeficientes de correlação genética e fenotípica. Entretanto, é possível constatar que as correlações do efeito temporário de ambiente, embora mais precisas (com menores erros-padrão), foram inferiores às demais.

As menores estimativas de correlação dos efeitos permanente e temporário de ambiente foram obtidas para P090d70\%, sendo as mesmas de $0,78(0,205)$ e $0,60(0,038)$, respectivamente.

Observa-se que as estimativas são mais altas entre PE pela projeção de menor porcentagem de lactações e P305d. Semelhantemente, as estimativas entre as PE pela projeção das lactações a partir de 211 ou 241 dias de lactação e a produção de leite até 305 dias foram mais altas.

As altas estimativas de correlação entre as produções obtidas pela projeção de uma porcentagem das lactações e a produção até 305 dias de lactação eram esperadas, já que a produção observada em parte da lactação está incluída na produção da lactação total. 


\section{Conclusões}

As herdabilidades das produções estimadas pela projeção de $10 \%$ das lactações a partir de $91,151 \mathrm{e}$ 211 dias ou pela projeção de $10,30,50$ ou $70 \%$ das lactações a partir de 241 dias foram semelhantes à obtida para a produção de leite até 305 dias.

Produções de leite estimadas pela projeção de 10 , 30,50 ou $70 \%$ das lactações a partir de $91,151,211$ ou 241 dias são altamente correlacionadas com a produção de leite até 305 dias.

\section{Referências Bibliográficas}

AURAN, T. 1976. Studies on monthly and cumulative monthly milk yield records. Acta Agric. Scand., (26):3-9.

BRASIL. Ministério da Agricultura. Secretaria Nacional de Produção Agropecuária. Portaria número 45 de 10 de outubro 1986. Normas Técnicas para Execução do Serviço de Controle Leiteiro em Bovídeos. Diário Oficial [da República Federativa do Brasil], Brasília, 124(198):15533-15535, 15 de out. 1986. Seção 1.

BOLDMAN, K.G., KRIESE, L.A., VAN VLECK, L.D. et al. 1995. A manual for of MTDFREML: a set of programs to obtain estimates of variances and covariances (DRAFT). Washington USDA, Agricultural Research Service. 115p.

DANELL, B. 1982. Studies on lactation yield and individual testday yields of Swedish dairy cow. II. Estimates of genetic and phenotypic parameters. Acta Agric. Scand., (32):103-114.

EVERETT, R.W., CARTER, H.W. 1968. Accuracy of test interval method of calculating dairy herd improvement association records. J. Dairy Sci., 51(12):1936-1941.

GENGLER, N., KEOWH. J.K., VAN VLECK, L.D. 1995. Various persistency measures and relationships with total, partial and peak milk yields. Rev. Bras. Gen., 18(2):237-243.

GONÇALVES, T.M., MARTINEZ, M.L., MILAGRES, J.C. 1997. Curva de lactação na raça Gir. 2. Influência dos fatores de meio ambiente, estimativas de repetibilidade e herdabilidade para os parâmetros da curva de lactação quadrática logarítmica. R. Bras. Zootec., 26(1):88-97.

KEOWN, J.F., VAN VLECK, L.D. 1971. Selection on test-day fat percentage and milk production. J. Dairy Sci., 54(2):199-203.

PANDER, B.L., HILL, W.G. 1993. Genetic evaluation of lactation yield from test day records on incomplete lactation. Lvstck. Prod. Sci., 37(1-2):23-36.

PANDER, B.L., HILL, W.G., THOMPSON, R. 1992. Genetic parameters of test day records of British Holstein - Friesian heifers. Anim. Prod., 55(1):11-21.

RAMOS, A. A. Estudo das características reprodutivas e produtivas de zebuínos leiteiros da raça Gir nos trópicos. Botucatu: UNESP, 1984. 224p. Tese (Livre Docência na Disciplina de Bovinocultura de Leite) - Universidade Estadual Paulista, 1984.
RIBAS, M., PEREZ, B. 1990. Monthly test day milk records and yield at 244 days. II. Genetic parameters in first lactation. Cuban J. Agric. Sci., 24(2):129-144.

RIBAS, M., PEREZ, B., GUZMAN, G. 1994. Monthly test-day yields and the extension of lactations in Cuba. Cuban J. Agric. Sci., 28(2):125-139.

SETHI, I.C., JAIN, J.P. 1993. Sire evaluation on partial records in dairy cattle. Ind. J. Anim. Sci., 63(8):869-872.

SOUZA, E.M. Estudos de fatores genéticos e no meio que influenciam o desempenho de rebanhos de Gir leiteiro. Viçosa: UFV, 1991. 103p. Dissertação (Mestrado em Melhoramento Genético) - Universidade Federal de Viçosa, 1991.

STATISTICAL ANALYSIS SYSTEM - SAS. 1995. User's guide: statistics. 5.ed., Cary. 1290p.

TANDON, P.K., HARVEY, W.R. 1984. Best linear unbiased prediction of sire breeding values from part lactations of daughters. J. Dairy Sci., 67(10):2399-2406.

VAN VLECK, L.D. p.1-31. Animal model for bull and cow evaluation. In: Large dairy herd management symposium. Gainesville, 1992.

VAN VLECK, L.D. 1964. Genetic parameters of five-month lactation records. J. Dairy Sci., 47(4):421-425.

VERNEQUE, R.S. Procedimentos numéricos e estimação de componentes de covariância em análise multivariada pelo método da máxima verossimilhança restrita: modelos mistos aplicados ao melhoramento animal. Piracicaba: ESALQ, 1994. 157p. Tese (Doutorado em Agronomia) - Escola Superior de Agricultura "Luis de Queiroz", 1994.

VERNEQUE, R.S., MARTINEZ, M.L., TEODORO, R.L. Avaliação genética de vacas e touros com base na produção de leite em diferentes estágios da lactação. In: REUNIÃO ANUAL DA SOCIEDADE BRASILEIRA DE ZOOTECNIA, 35, 1998, Botucatu. Anais ... Botucatu: SBZ, 1998. p.255-257.

WILMINK, J.B.M. 1987. Efficiency of selection for different cumulative milk, fat, and protein yields in first lactation. Lvstck. Prod. Sci., (17):211-224.

ZAHED, S.M., KHALIL, M.H., SOLIMAN, A.M. 1997. Comparison between efficiency of part- e complete-lactation record in progeny testing Fleckvieh bulls. Egyp. J. Anim. Prod., 34(1):11-26. CD-ROM. CAB Abstracts 01/1996 07/1998.

Recebido em: 01/07/99

Aceito em: 30/11/99 